\title{
Thema Kommunikation auf dem physiokongress
}

\author{
Der physiokongress 2008 kommt! „Kommunikation“, „Gesundheitsförderung und \\ Prävention“ und „Langzeitpatient“ werden vom 12.-14. Juni 2008 in Fellbach bei \\ Stuttgart die großen Themen sein. Lesen Sie hier, was die Keynotespeaker zum \\ Thema Kommunikation zu sagen haben.
}

D e Auftaktveranstaltungen zu den vielen interessanten Vorträgen des physiokongresses bilden dieses Jahr die sogenannten Keynote-Referate. Diese sollen die Kongressteilnehmer einmal pro Tag versammeln und sie den Kongress gemeinsam erleben lassen. Die Vortragenden, Keynotespeaker genannt, sind Experten, die das jeweilige Schwerpunktthema umfassend beleuchten und deshalb alle Kongressteilnehmer ansprechen. In einer dreiteiligen Serie stellt physiopraxis hier

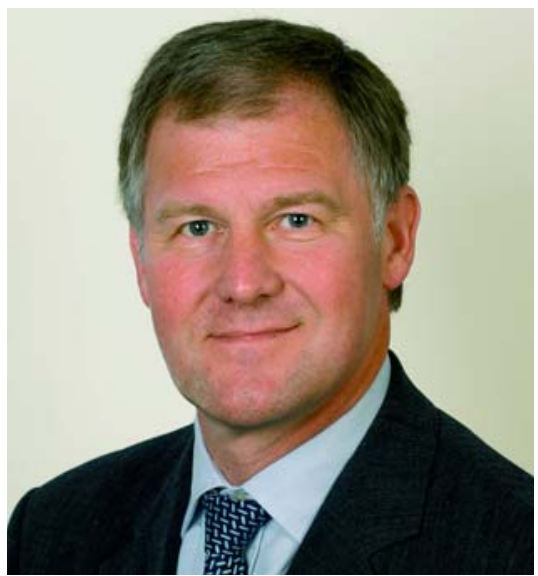

Dr. Michael Wolf ist Psychologe und Informationswissenschaftler. Er hat sich mit der Frage befasst, wie sich die Kommunikation zwischen Personen oder Gruppen optimieren lässt, um bestmögliche Resultate zu erreichen. Dazu hat er Trainingsprogramme entwickelt und Vorträge gehalten. Gegenwärtig arbeitet er im Risikomanagement bei einer Großbank in der Schweiz. Außerdem ist er für das Qualitätsmanagement bei der Akademie für integrative Physiotherapie und Trainingslehre (AfiPT) in GrenzachWyhlen verantwortlich. Dort kümmert er sich um die praxisgerechte Aufbereitung von Studien. alle sechs Keynotespeaker und die Themen ihrer Referate vor. Cornelia Schneider und Dr. Michael Wolf machen in dieser Ausgabe den Anfang mit dem Thema Kommunikation.

Wie wichtig die Kommunikation in der Physiotherapie ist, zeigt eine Studie von Forschern der Universität Turin, Italien: Diese haben herausgefunden, dass Placeboeffekte Schmerzen wirklich lindern können. So weit, so gut. Eine besonders starke Placebowirkung haben die Forscher beobachtet, wenn der Therapeut dem Patienten sehr vertrauensvoll und empathisch erschien. Das Verhältnis zwischen dem Physiotherapeuten und dem Patienten beeinflusst also das Therapieresultat, und die Kommunikation der beiden ist die Grundlage dieser Beziehung. Dass man miteinander spricht, heißt aber nicht immer, dass man sich versteht. Michael Wolf spricht auf dem physiokongress darüber, wie wir Hürden in unserer Kommunikation erkennen können.

\section{> Welche Rolle spielt Kommunikation in Ihrem Berufsalltag?}

In meiner Tätigkeit als Risikomanager bin ich für ein Projekt verantwortlich, in dem circa 150 Personen verschiedenster Fachrichtungen über zwei Jahre intensiv zusammenarbeiten. Einer funktionierenden Kommunikation kommt hierbei eine erfolgsentscheidende Bedeutung zu. In der Akademie für integrative Physiotherapie und Trainingslehre werden neuste wissenschaftliche Erkenntnisse gesammelt und aufbereitet. Dort bin ich dafür verantwortlich, dass dieses Wissen strukturiert und so dargestellt wird, dass es ein breiter Kreis von Anwendern in der täglichen Praxis umsetzen kann.

\section{> Warum sollte man überhaupt über Kom- munikation reden?}

In unserm Alltag gehen wir häufig ganz selbstverständlich davon aus, dass unsere Bemühungen, zu kommunizieren, erfolgreich sind. Betrachtet man, was alles auf dem Weg von der Absicht, etwas zu kommunizieren, und dem, was beim Empfänger ankommt, passieren kann, wird deutlich, dass das Risiko, dass hierbei etwas verloren geht, größer ist, als wir intuitiv annehmen.

\section{$>$ Wie entstehen kommunikative Hürden? \\ Kommunikation wird von mehreren Faktoren beeinflusst: unter anderem von der Akustik, der Aufmerksamkeit des Empfängers, seiner Bereitschaft, eine spezielle Botschaft aufzu- nehmen, und davon, wie dieser Empfänger}

Gesagtes mit seinem Vorwissen abgleicht. Somit gibt es viele Größen, welche Kommunikation stören oder unmöglich machen.

> Wie kann ich prüfen, was von einer Botschaft angekommen ist?

Was von einer Botschaft angekommen ist, wird zum Beispiel durch Handlungen, die auf eine Instruktion folgen, prüfbar. Häufig gibt man sich gerne mit einem "Ja“ als Antwort des Empfängers auf die Frage: „Haben Sie das verstanden?“ zufrieden. Immer, wenn der Angesprochene glaubt, etwas verstanden zu haben, wird er der Frage zustimmen. Wenn er aber etwas anders verstanden hat, als vom Sender beabsichtigt, so bleibt dieses Missverständnis so natürlich unentdeckt.

\section{$>$ Welche kommunikativen Herausforderun-} gen stellen sich in der Physiotherapie?

Nehmen wir zum Beispiel die aufrechte Körperhaltung: Man sagt dem Patienten, welche Position er einnehmen soll, der Therapeut sieht das Ergebnis und folgert darauf, dass die Anweisungen verstanden worden sind. Damit fällt die Erfolgskontrolle der Kommunikation einfach und eindeutig aus. Diese Vorgehensweise ist bei einer Beckenbodenübung jedoch nicht geeignet, weil die Instruktion keine sichtbare Veränderung nach sich zieht. Der erste Schritt zu einer verbesserten Kommunikation liegt darin, dass uns bewusst wird, dass wir mit Worten längst nicht so viel bewirken, wie wir denken!

Das Gespräch führte Adriana Pfisterer. 
W enn wir miteinander kommunizieren - und in der Physiotherapie tun wir das permanent -, nutzen wir immer mehrere Kommunikationskanäle gleichzeitig. Durch verbale Äußerungen vermitteln wir Informationen, die wir durch Gestik, Mimik und Stimme (zum Beispiel die Lautstärke oder die Tonlage), durch Berührungen, die Distanz zum Patienten, unsere äußere Erscheinung und

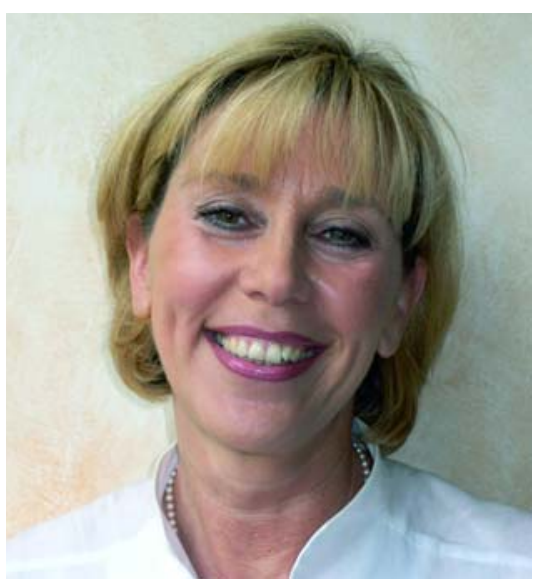

Cornelia Schneider ist Physiotherapeutin und Psychologin. Sie leitet eine Praxis für Physiotherapie, ist geschäftsführende Gesellschafterin der Gesellschaft für Gesundheitspflege und interdisziplinäre Weiterbildung in Homburg/Saar (GGW) und bietet Seminare für Physiotherapeuten und andere Berufsgruppen zu verschiedenen Aspekten der Kommunikation an.

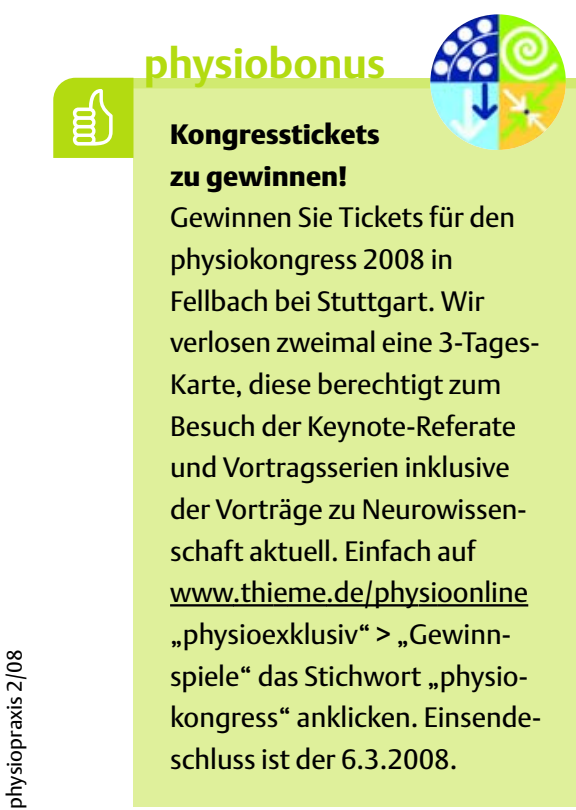

das umgebende Ambiente stärken oder schwächen. Unser Körper sendet Signale aus, die der Patient im Unterbewusstsein wahrnimmt. Und der Patient vermittelt uns durch seine Körpersprache Informationen, die er nicht ausspricht, denn jede Gefühlsregung hat eine Entsprechung im Körperlichen, sei es in vegetativen oder motorischen Funktionen. Das kann subtil und kaum wahrnehm- bar sein, wie zum Beispiel ein kurzes Niederschlagen der Augenlider bei einer Lüge. Umgekehrt wirkt sich motorische Aktivität auch auf emotionale und geistige Prozesse des Menschen aus, das zeigen Befunde aus den Neurowissenschaften. Cornelia Schneider wird das Wissen der Kongressbesucher über Körpersprache in der Physiotherapie und der Prävention erweitern.
> Gab es ein Schlüsselerlebnis, das Sie dazu motivierte, sich verstärkt mit der Thematik auseinanderzusetzen?

Bereits in den 70er-Jahren habe ich festgestellt, dass Haltungsveränderungen in der Therapie trotz intensiver physiotherapeutischer Bemühungen nicht internalisiert werden. Der Erfolg einer Behandlung korrespondiert immer mit der inneren Haltung eines Patienten. So sprach ich damals mit einem Neurologen und stellte ihm folgenden Sachverhalt dar: Rückenschulen, wie sie von Physiotherapeuten vermittelt werden, können niemals erfolgreich sein, da sie die emotionale Komponente vernachlässigen. Die Antwort war befremdlich, das sei „alles nur Folklore“. Seit den 90er-Jahren gibt es jedoch wissenschaftliche Belege, durch die eindeutig nachgewiesen ist, dass Bewegung emotional gesteuert wird.

> Wie gehen Physiotherapeuten mit den Informationen der Körpersprache um?

Das Wissen über Körpersprache und entsprechende Erfahrungen sind durchaus vorhanden. Problematisch scheint eher die Anwendung in der Therapie, weil die Therapeuten dazu kein Handlungsschema besitzen. Das Bewegungssystem ist einerseits Instrument zur Anpassung an die Umwelt und andererseits eine Möglichkeit, mit der Umwelt zu kommunizieren. Physiotherapeuten betrachten jedoch häufig nur die funktionelle Ebene.

> Es gibt Wissenschaftler, welche sich mit der räumlichen Konstellation von Kommunikationspartnern beschäftigen, man nennt das Proxemik. In der Proxemik teilt man die Abstände zwischen Personen in intime, persönliche, soziale und öffentliche Distanzen ein. Ist Proxemik auch ein wichtiges Thema in der Physiotherapie?

Proxemik hat in der Physiotherapie einen besonderen Stellenwert, weil wir uns bei jeder Behandlung mit Nähe und Distanz auseinandersetzen müssen. Berührung wirkt sich auf
Körperfunktionen aus, das ist nachgewiesen durch biochemische Parameter. Damit hat sie auch einen Einfluss auf die Emotionen der Patienten. Physiotherapeuten müssen sich bewusst sein, dass die Bereitschaft der Patienten zur Berührung erst geschaffen werden muss. So scheint es banal, ist aber notwendig, den Patienten nach seiner Erlaubnis zur Berührung zu fragen.

\section{>Warum fällt es Physiotherapeuten so} schwer, mit Körpersprache umzugehen?

Die Arbeit mit der Körpersprache löst unterschiedliche Reaktionen aus. Physiotherapeuten haben Angst davor, mit den aufkommenden Gefühlen der Patienten umzugehen. Sie brauchen nicht nur die Fähigkeit, zielgerichtet Fragen zu stellen und Aussagen zu reflektieren, sondern auch die Kompetenz, Emotionen der Patienten aufzufangen.

\section{$>$ Wie können Physiotherapeuten ihr Wissen} über Körpersprache in anderen Bereichen einsetzen?

Das Wissen über Körpersprache bietet neue Arbeitsfelder für Physiotherapeuten. Menschen in Körpersprache zu schulen wird in unterschiedlichen Bereichen angestrebt. Manche Menschen möchten sich einfach wohler fühlen, selbstbewusster sein, Charisma entwickeln und fragen nach Möglichkeiten, dieses durch ihren Körper zu signalisieren. Hier geht es mehr darum, zu erfassen: Was muss ich an meiner Gewohnheitshaltung ändern - funktionell oder emotional -, um die Körpersprache wieder möglich zu machen? Andere Berufsgruppen setzen Körpersprache ganz gezielt ein, um ihrer Position mehr Nachdruck zu verleihen, Menschen besser zu motivieren oder einfach um mehr persönlichen und privaten Erfolg zu haben. Es ist daher nicht erstaunlich, dass ich die meisten meiner Seminare im Managementbereich gebe und bisher nur wenige im Gesundheitswesen.

Das Gespräch führte Adriana Pfisterer. 